\title{
Knowledge and Attitude Regarding Cigarette Smoking Among UG Students
}

\author{
Kevin Silas Christian \\ (Assistant Professor, Department of Medical Surgical Nursing, Sumandeep Nursing College, India)
}

\begin{abstract}
Today's smoking culture includes a subpopulation of smokers called "social smokers". Many college students define "social smokers" as those who use tobacco in more social activities, rather than using tobacco on a regular basis. Social smokers don't believe that they are addicted or worried about the social acceptability of their smoking habits.
\end{abstract}

Objectives: To assess the knowledge and attitude of cigarette smoking use among students and to find out the association between knowledge and attitude and their association with demographic variables.

Materials and methods: Non-experimental descriptive design was used to select 100 samples. Purposive sampling is used in which handpicking of subjects were done. Questionnaires and 3 point likert scale was used to assess the knowledge and attitude of the samples. Descriptive and Inferential Statistics was used to analyze the data.

Results: There is no association between knowledge and attitude regarding cigarette smoking. Association between knowledge regarding cigarette smoking with age and mother's education of the participants was found. There is significant association between father's education and occupation and type of family with attitude of the students.

Conclusion: There is a probability of having role models from the family regarding the habit of cigarette smoking. It seems that there is a need for the health care providers for developing programs for eradication of cigarette use among the students.

Keywords: cigarette smoking, knowledge, attitude, undergraduate students.

\section{Introduction}

Tobacco-related deaths have nearly tripled around the world in the past decade. If trends continue, 1 billion people will reportedly die from tobacco use and exposure during the 21 st century -1 person every 6 seconds ${ }^{1}$. Today's smoking culture includes a subpopulation of smokers called "social smokers". Although there may be different explanations of what a social smoker is, many college students define "social smokers" as those who use tobacco in more social activities and find it essential for socializing, rather than using tobacco on a regular basis, dictated by nicotine dependence. Social smokers don't believe that they are addicted to smoking, or worried about the social acceptability of their smoking habits ${ }^{2}$. The overwhelming majority of smokers begin tobacco use before they reach adulthood. Among those young people who smoke, nearly one-quarter smoked their first cigarette before they reached the age of ten. Several factors increase the risk of youth smoking. These include tobacco industry advertising and promotion, easy access to tobacco products, and low prices. Peer pressure plays an important role through friends' and siblings' smoking. Other risk factors associated with youth smoking include having a lower self-image than peers, and perceiving that tobacco use is normal or "cool". Many studies show that parental smoking is associated with higher youth smoking. ${ }^{3}$

\section{Need For The Study}

Philip Morris Companies Inc. is an international cigarette company which believes on knowing the attitude of teenagers and their customers to make their company more efficient. Thus, here comes the job of health professional to mould the behavior and to bring health awareness by assessing knowledge and attitude of the cigarette smoker and which can be used by health authorities under National Tobacco Control Program. In view of the high prevalence of tobacco use in the country, there should be a national effort to prevent any further increase in the prevalence of tobacco use, especially among the vulnerable groups such as females, youth and children. This study helps in understanding and focusing on the knowledge and attitude aspects of undergraduate students regarding cigarette smoking. One of the study was conducted at Irbid, Jordan in the year 2013. Only $10.4 \%$ of the students believed that a smoking female student has more friends than a non-smoking female student. However, the rate of smoking students who believed that a smoking female has more friends was significantly higher $(P<0.05)$ than that of non-smoking students. Here however, the rate of nonsmoking students that believed a smoking male has more friends was actually higher than that of smoking students. 
Smoking students who believed that a smoking male has a strong personality were almost twice as much as nonsmokers ${ }^{4}$.

In view of the high prevalence of tobacco use in the country, there should be a national effort to prevent any further increase in the prevalence of tobacco use, especially among the vulnerable groups such as females, youth and children. There should also be targeted programs addressing different types of tobacco use and different user groups with special focus on cessation. There is a need to further strengthen the implementation of Cigarettes and Other Tobacco Products (Prohibition of Advertisement and Regulation of Trade and Commerce, Production, Supply and Distribution) Act, 2003, at national, state and sub-state levels. Establishment of a comprehensive implementation and regulatory structure at the national and state level is required ${ }^{5}$. Increased tobacco control education and related efforts are needed as is further research in determining the factors or influences that shape the significant differences found. ${ }^{6}$

\section{Statement Of Problem}

To assess the knowledge and attitude regarding cigarette-smoking among undergraduate students of selected colleges of Pune city.

\section{Objectives}

1.1. To assess the knowledge of cigarette smoking use among students.

1.2. To assess the attitude of students towards cigarette smoking.

1.3. To find out the association between knowledge and attitude regarding cigarette smoking.

1.4. To find out the association between knowledge, attitude with the demographic variables.

\section{Operational Definition:}

2.1 Assess - It refers to the finding of the knowledge and attitude about cigarette smoking in undergraduate students.

2.2. Knowledge - It refers to the response of the undergraduate students regarding cigarette smoking according to structured questionnaire.

2.3 Cigarette smoking - It refers to the habit of inhalation of smoke through cigarettes which is done actively at regular/irregular interval.

2.4 Undergraduate - It refers to the student who is studying under Bachelor's degree under any course.

\section{Assumptions}

3.1 The undergraduate students will have some knowledge on cigarette smoking, its ill effects on human body.

3.2 The knowledge about cigarette smoking may influence on the attitude of the student.

3.3 There will be some correlation between some of the demographic variables and knowledge and attitude of the students.

\section{Hypothesis}

4.1 $\mathbf{H}_{0}$ - There is no significant association between knowledge and attitude regarding cigarette smoking among undergraduate students of selected colleges of Pune city.

4.2 $\mathbf{H}_{1}$ - There is significant association between knowledge and attitude regarding cigarette smoking among undergraduate students of selected colleges of Pune city. 


\section{Conceptual framework}

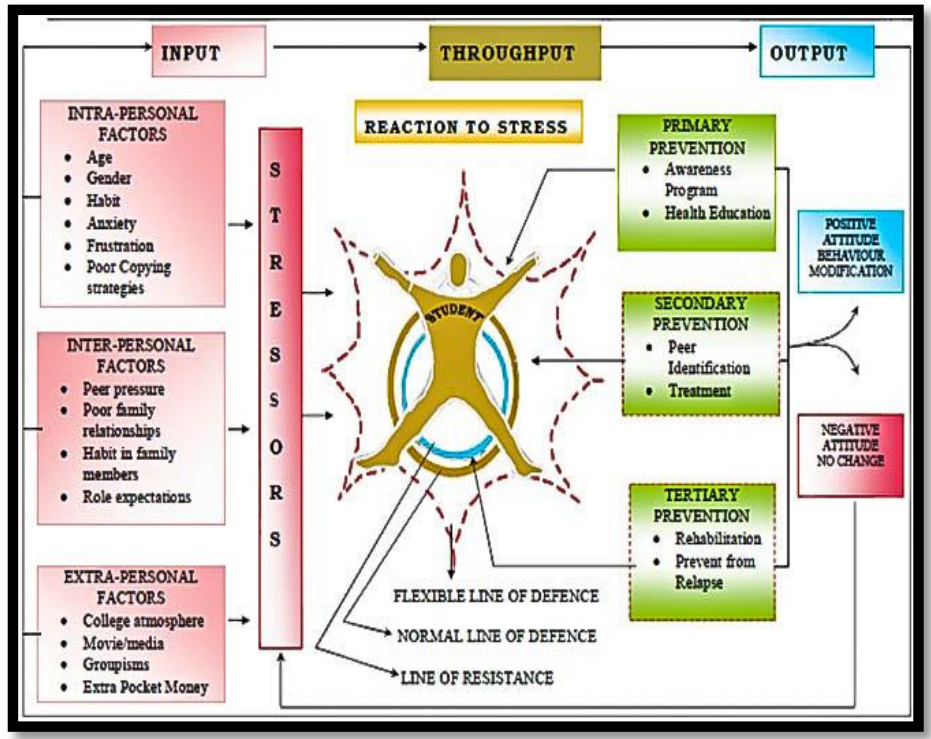

Fig 1:- Conceptual framework - Betty Neuman System Model

\section{Methodology}

This chapter deals with a brief description of methodology adopted for the study. The contents included in this research are research approach, research design, the setting, the sampling and sampling technique, development and description of tool, data collection procedure and plan for analysis for the present study.

\section{Research Design}

Research design will be non-experimental descriptive design to find out the knowledge and attitude regarding cigarette smoking among undergraduate students at selected colleges.

It is a type of survey in which investigation is done with the help of self-report data with the purpose of describing populations on some variable or variables of interest. ${ }^{52}$

\section{Research Approach}

In this study, researcher has used descriptive survey approach. Quantitative research is concerned with objectivity, tight controls over the research situation and the ability to generalize the findings. ${ }^{50}$

In descriptive studies, phenomena are described or the relationship between variables is examined. In such studies, hypotheses are tested. ${ }^{51}$

Quantitative research is directed at the discovery of relationships and cause and effect. Methods used are based on the scientific method of inquiry. Quantitative refers to measurement and analysis of causal relationships between variables at a particular point in time.

\section{Variables}

Independent Variable

Cigarette smoking is the independent variable in this study.

\section{Dependent Variable}

Knowledge and attitude are the dependent variable in this study.

\section{Extraneous Variable}

In the present study it refers to the selected demographic variables such as age, gender, educational status of father and mother, occupational status of father and mother, education, family type, income, source of information, etc.

\section{Setting of the Study}

This study was undertaken in selected colleges of Pune city after due permission and ethical clearance.

The setting was selected on the basis of:-

$>$ Geographic proximity.

$>$ Feasibility of conducting study. 
$>$ Availability of the sample.

$>$ Ethical clearance

$>$ Availability of samples.

Population

The population comprised of all undergraduate cigarette smoker students of Pune city.

\section{Sample and Sampling Technique}

Sample is the small proportion of population selected for observation and analysis. Sampling is the process of selecting representative units of a population for study in a research process. In this study, nonprobability sampling method is used. The sample elements are chosen from the population by nonrandom methods. Purposive sampling (judgmental sampling) is used in which handpicking of subjects are done.

\section{Sample size}

Sample size consists of 100 undergraduate students of selected colleges of Pune city.

Criteria for Selection of Samples

The criteria that specify population characteristics are referred as eligibility criteria or inclusion criteria.

\section{Inclusion Criteria}

$\checkmark$ Undergraduate student

$\checkmark$ Cigarette-smoker

$\checkmark \quad$ Willing to participate

$\checkmark$ Those who understands English

\section{Exclusion criteria}

* Post-graduate student

* Student of college other than Pune city

\section{Development of tool}

The tool was developed based on:

$\checkmark$ Past clinical experience of the student investigator

$\checkmark$ Related review of literature (books, journals, reports, articles, published and unpublished studies) were reviewed and used to develop the tool.

$\checkmark$ Guidance and consultation with the guide and subject experts.

$\checkmark$ Objectives of the study and blue print.

\section{Description of tool}

Structured questionnaire was used as a research instrument. The tool is divided into following parts:

A. Part I: Baseline Proforma

B. Part II: Assessment of Knowledge

C. Part III: 3 point Likert Scale to assess attitude

\section{Part I: Baseline Proforma}

It contains items such as age, gender, education, education of father and mother, occupation of father and mother, family type, family income, family member with habit of smoking, other habit apart from cigarette smoking, kind of cigarette smoked, any physical complaints, awareness of ill-effects of smoking, source of information, etc.

\section{Part II: Assessment of Knowledge}

It is divided into three sub-divisions:

a) Assessment of knowledge regarding tobacco

b) Assessment of knowledge regarding ill effects of cigarette smoking

c) Assessment of knowledge regarding de-addiction of cigarette smoking

\section{Part III: 3 point Likert scale to assess attitude}

After reviewing literature and having discussion with experts, 20 statements were made to assess attitude of the student regarding cigarette smoking. 3 point Likert scale is used to provide the responses of the student. 


\section{Data Analysis}

Table 1:- Association between knowledge and attitude score with selected demographic variables

\begin{tabular}{|l|l|l|l|l|l|l|}
\hline Sr. No. & Baseline Proforma & $\mathbf{d F}$ & \multicolumn{2}{|l|}{ p value } & \multicolumn{2}{l|}{ Association } \\
\cline { 4 - 7 } & & & $\mathbf{K}$ & $\mathbf{A}$ & \multicolumn{2}{l}{ K } \\
\hline 1 & Age & 8 & .002 & .410 & Yes & No \\
\hline 2 & Gender & 2 & .131 & .662 & No & No \\
\hline 3 & Education & 12 & .415 & .669 & No & No \\
\hline 4 & Father's Education & 10 & .303 & .011 & No & Yes \\
\hline 5 & Mother's Education & 10 & .003 & .436 & Yes & No \\
\hline 6 & Father's Occupation & 12 & .344 & .028 & No & Yes \\
\hline 7 & Mother's Occupation & 6 & .188 & .444 & No & No \\
\hline 8 & Family type & 6 & .050 & .024 & No & Yes \\
\hline 9 & Family income & 6 & .834 & .400 & No & No \\
\hline 10 & Family member with smoking habit & 2 & 0.786 & .582 & No & No \\
\hline 11 & Who introduced & 6 & .425 & .335 & No & No \\
\hline 12 & Age of initiation & 6 & .347 & .057 & No & No \\
\hline 13 & How often smoke & 8 & .672 & .167 & No & No \\
\hline 14 & Learnt about effects of smoking & 8 & .112 & .448 & No & No \\
\hline 15 & Aware about all ill-effects & 2 & .092 & .117 & No & No \\
\hline
\end{tabular}

Note:- K - Knowledge, A - Attitude, p value $<\mathbf{0 . 0 0 5}$

Table 2:- Chi-square for knowledge and attitude association

\begin{tabular}{|l|l|l|l|}
\hline Formula & Value & df & $\begin{array}{l}\text { Asymptotic. Sig. (2- } \\
\text { sided) }\end{array}$ \\
\hline Pearson Chi-Square & $5.451(\mathrm{a})$ & 4 & .244 \\
\hline Likelihood Ratio & 5.584 & 4 & .232 \\
\hline $\begin{array}{l}\text { Linear-by-Linear } \\
\text { Association }\end{array}$ & .001 & 1 & .976 \\
\hline McNemar-Bowker Test & 4.000 & 3 & .261 \\
\hline N of Valid Cases & 100 & & \\
\hline
\end{tabular}

Asymptotic Significance value is greater than 0.05 hence there is independency between knowledge and attitude variable. The chi-square value is 0.244 which is greater than 0.05 . Thus null hypothesis is accepted. And one can say that there is no significant association between knowledge and attitude of participants regarding cigarette smoking.

There is no association between knowledge and attitude of the participants regarding cigarette smoking $(\mathrm{p}=0.244)$. $\mathrm{p}$ value is greater than 0.05 . So null hypotheses is accepted.

There is significant association between knowledge regarding cigarette smoking and age of the participants $(\mathrm{p}=0.002)$. There is significant association between mother's education level and knowledge regarding cigarette smoking in the participants $(\mathrm{p}=0.03)$. There is significant association between father's education and attitude of the students regarding cigarette smoking $(\mathrm{p}=0.011)$. There is significant association between father's occupation and attitude of the students regarding cigarette smoking $(\mathrm{p}=0.028)$. There is significant association between type of family and attitude of the students regarding cigarette smoking $(\mathrm{p}=0.024)$.

\section{Conclusions}

There is no correlation between knowledge and attitude regarding cigarette smoking among undergraduate cigarette smoker students. There is a probability of having role models from the family regarding the habit of cigarette smoking. Awareness is present in the students but the behavior is still not healthy. In spite of knowledge, behavior modification becomes difficult in this technological and innovative world. Cigarette smoking incidence is seen more in higher middle class and higher class. Type of family has association with the knowledge of the cigarette smoker students regarding cigarette smoking. Age has strong association with the knowledge of the student regarding cigarette smoking. 31\% believe that smoking must be banned everywhere in spite of being a smoker. This implies the easy availability of the cigarette had impact on their habit of cigarette smoking. $16 \%$ of the respondents have a wrong attitude that passive smoking is not injurious to health. $69 \%$ of the respondents have positive attitude that one should not encourage others for smoking. $11 \%$ of the students are frightened about their habit of smoking and have social stigma for smoking. More than half of the students $(52 \%)$ believe that they can leave the habit of smoking. $64 \%$ of the smokers have positive attitude that they don't like to smoke in 'no smoking zone' area. $80 \%$ of the smokers agree on the statement 'Money spent on cigarette can be spent on buying nutritious food', More than half of the students $(57 \%)$ regret on their habit of smoking justifying the need of de-addiction. 


\section{References}

[1]. http://www.who.int/gho/ncd/mortality_morbidity/chronic_respiratory_diseases_text/en/index.html

[2]. http://www.who.int/tobacco/publications/surveillance/fact_sheet_mortality_report.pdf

[3]. http://articles.timesofindia.indiatimes.com/2009-08-27/india/28156183_1_female-smokers-tobacco-industry-world-lung-foundation (cited on 25/09/2012)

[4]. Knowledge, attitude and practice of university students towards smoking in Irbid, Jordan, Journal of Public Health and Epidemiology Vol. 5(1), pp. 29-36, January 2013

[5]. http://www.who.int/tobacco/surveillance/en_tfi_india_gats_fact_sheet.pdf

[6]. Knowledge, Attitude and Practice of Smoking among University Students of Allied Health Sciences in Japan, Asia Pacific Journal of Public Health, Volume 12, Issue 1, 2000, pg no. 17-21 